\title{
Monitoring prescribing: GPs' use of Prescribing Analysis and Cost (PACT) data
}

Miren I. Jones, Sheila M. Greenfield, Sue Jowett Department of Primary Care and General Practice, University of Birmingham, Birmingham, UK, Amanda Nayak Department of Accounting and Finance, Birmingham Business School, University of Birmingham, Birmingham, UK, Fiona A. Stevenson Department of General Practice, GKT School of Medicine, London, UK and Colin P. Bradley Department of General Practice, University College Cork, Cork, Ireland

\begin{abstract}
Prescribing Analysis and Cost (PACT) data are sent to all GPs to assist them in monitoring their prescribing. Although the quarterly Standard Reports contain a great deal of information it is not known how GPs make use of it. This paper reports on two linked studies which explore GPs' views on PACT Standard Reports and PACT catalogue data. In the first study, interviews were carried out with $21 \mathrm{GPs}$ in 16 practices selected according to criteria related to their prescribing budget. The interview schedule included questions on how the Standard Reports were used and the amount and ease of understanding of the information provided, and on use of PACT Catalogue data. The second study was a questionnaire survey of the 1047 practices in the West Midlands region in which GPs were asked how they used PACT data. Analysis of the interviews showed that GPs' views on the Standard Report varied widely although most found it helpful. Many GPs used the data in a limited way and only used a small part of them. Single-handed GPs and small practices were least likely to make use of PACT data and few practices analysed the data in any depth. Most respondents said that comparing their costs with health authority and national averages was useful for considering where to make changes in their prescribing. GPs indicated they would like the report to include some interpretation of the data with suggestions for changes which could be made. The regional survey confirmed that PACT Standard Reports are only used in a limited way and how they are used varies with practice size. We conclude that PACT Standard Reports were rarely used to analyse prescribing in any depth and GPs need more support in making better use of the data.
\end{abstract}

Key words: PACT; GP; prescribing

\section{Introduction}

General practice prescribing costs increased by an average of 9\% per year from 1987 to 1997 (Prescription Pricing Authority 1997) and there has been increasing pressure on individual general practitioners (GPs) to control their prescribing costs. There is now new and greater formal organizational pressure from Primary Care Groups (PCGs) which, together with a new funding for-

Address for correspondence: Dr M. Jones, Department of Primary Care and General Practice, University of Birmingham, Edgbaston, Birmingham B15 2TT, UK. Email: M.I.Jones@bham.ac.uk

(C)Arnold 2002 mula, were introduced in April 1999 and have a cash-limited budget. Many PCGs are now merging to form PCTs with more responsibilities and greater control of the budget. A number of external methods for cost control have been introduced including fundholding (Bradlow and Coulter, 1993), the prescribing incentive scheme (Bateman et al., 1996) and indicative prescribing budgets (Greenhalgh, 1998; Majeed and Head, 1998). One tool which, in theory, allows GPs to look critically at the nature and cost of their prescribing is PACT (Prescribing Analysis and Cost) data but little is known about how GPs use it.

PACT data, produced by the Prescription Pricing Authority (PPA), was introduced in 1988 and 
changed to its present form in 1994 (Prescription Pricing Authority, 1994). PACT Standard Reports are sent quarterly by the PPA to individual GPs in paper format. The Standard Report provides statistics comparing the prescribing activities of the practice as a whole and some data on individual GP prescribing. The statistics are compared with the Health Authority (HA) equivalent and a national equivalent, and include the change from the previous year, thus allowing a GP to make basic performance comparisons (Bevan, 1996). A PACT catalogue is available on request from the PPA and provides a complete breakdown of prescribing at GP or practice level down to individual drugs or BNF groups according to the level of detail requested.

A thorough analysis of PACT data, therefore, can potentially yield a great deal of useful information which could be used to monitor individual and practice prescribing patterns. However, one study found no evidence to support the view that PACT had increased awareness among GPs of the cost of their prescribing decisions (Ryan et al., 1992). Majeed et al. (1997) have suggested that many GPs may not make much use of PACT. As the PACT Standard Report is 12 pages (including the centre page report on a special topic) and the catalogue can be very long, low usage might simply be due to time constraints or to the need for training in analysis and interpretation in order for GPs to maximize their use of the data.

No study exists of how GPs use PACT since the current format was introduced in 1994. This paper reports the results of two linked studies which asked GPs about their methods of controlling prescribing costs and included questions on their attitudes to and use of PACT data. The first of these was a qualitative study of 16 practices selected according to criteria related to their prescribing budget. The results were used to inform the development of a questionnaire for the second study, which was a survey of all the practices in the West Midlands region.

\section{Methods}

\section{Study 1}

This was designed with a purposive sample recruited from the extremes of the selection criteria (practices with either a high- or low-budget allo- cation relative to the HA average) in order to encompass a wide range of views (Mays and Pope, 1995). Sixteen practices were selected from within Birmingham Health Authority to include eight with a 'high' and eight with a 'low' prescribing budget allocation relative to the HA average (Figure 1). Initially, two fundholding and two nonfundholding practices were selected for each over and underspending subgroup, but at the time of the interviews there were 10 fundholding and six nonfundholding practices due to changes in fundholding status. A total of 44 practices were approached until 16 practices had been recruited into the appropriate categories.

In-depth interviews were carried out with 21 GPs in the 16 practices between June 1997 and February 1998. One practice (P10) employed a pharmacist who was also interviewed. All the interviews were recorded and transcribed. The main analysis was carried out by authors M.J., S.G. and F.S. The transcripts were analysed by the technique of charting (Bryman and Burgess, 1994), which involved repeatedly reading the transcripts and independently selecting and reorganizing responses according to themes. Developing themes were then discussed and further refined in meetings of all the authors. Further details of the interviews and analysis are described elsewhere (Stevenson et al., 1999). The semi-structured interview schedule included specific questions on the PACT Standard Report and focused on four areas: amount of information, ease of understanding of the information provided, how it was used and how helpful it was perceived to be. The GPs were also asked about their use of PACT Catalogue data.

\section{Study 2}

The themes resulting from the interviews were used to develop a four page postal questionnaire containing both open (free text) and closed (tick box) questions on a range of issues which might have an influence on prescribing budgets. The themes covered were: perceived influences on practice drug spending, such as patient groups, ethnic mix, environmental factors and prevalence of chronic disease; external sources of prescribing advice and information; use of PACT data; measures taken to reduce prescribing costs; and sociodemographic data. The questionnaire included a list of 11 sections of the PACT Standard Report and respondents were asked to indicate which sec- 


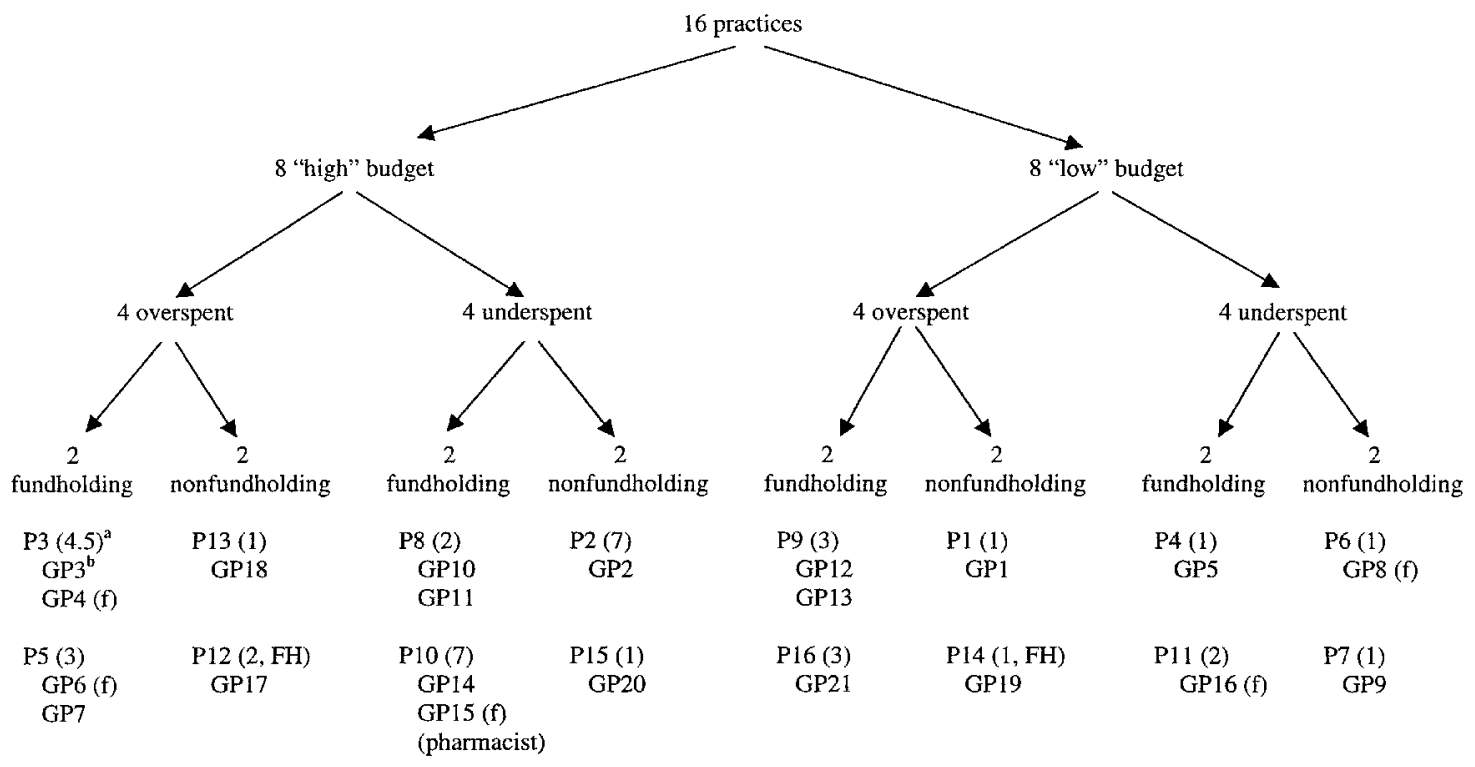

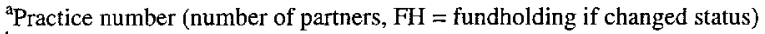

${ }^{\mathrm{b}} \mathrm{GP}$ number ( $\mathrm{f}=$ female)

Figure 1 Selection of practices for Study 1 interviews

tions they used and which they found most useful in helping them to analyse their prescribing. Respondents were also asked if they had ordered the PACT Catalogue in the last 2 years and how they had used it, and to indicate if their practice costs were above or below the HA equivalent based on their Standard Report for the quarter ending December 1997. The questionnaire was sent to the senior partner in all 1047 practices in the West Midlands Region. Two further mailings were sent to non-responders.

Responses to open questions were analysed independently by two of the researchers who selected and reorganized the responses according to themes. The data were coded and analysed by SPSS 8 for Windows. Nonparametric tests were used to analyse the data as appropriate. Statistical tests were employed for the comparison of frequencies ( $\chi^{2}$ test), trends in proportions ( $\chi^{2}$ for trend) and comparison of independent groups (Mann-Whitney $U$ test). For the purposes of analysis, practices were divided into four groups according to size. Practices were defined as small (1-2 partners), medium (3-4 partners) and large (5 or more partners).

\section{Results}

\section{Study 1 (interviews)}

The overall use of PACT Standard Reports was limited both in the amount and the depth in which they were used. The way in which PACT was used varied with practice size, with larger practices generally using it in a more systematic way. GPs in smaller practices felt that they already knew well enough their own prescribing. There was also some confusion among GPs between the quarterly Standard Reports and monthly budget statements, which are also prepared by the PPA; some GPs discussed these interchangeably which suggested a superficial use of the data rather than a detailed analysis. The main themes arising from the interviews, with examples, are summarized in Box 1 . 
Box 1 Summary of themes from interviews

- Theme 1 - How PACT Standard Reports are used:

'...but for us we know what is happening more or less in a small practice. I look at it I don't analyse it.' GP8 (low, under, $N F H, 1)^{\mathrm{a}}$

'[pharmacist] has them ... and discusses them but we don't. I don't keep it or look at it regularly or check it.' GP14 (high, under, FH, 7)

'Yes, we do [a regular analysis] then we discuss it to see what is going on, if we can improve it.' GP17 (high, over, $\mathrm{FH}, 2)$

'I look through it when I want to but I will not do an analysis.' GP5 (low, under, FH, 1)

'we do all read our PACT data and have made efforts, though on a kind of as and when basis rather than a formal way. ... I don't always use the PACT stuff in any great depth because I haven't got time.' GP3 (high, over, $\mathrm{FH}, 4.5)$

'we look at our figures and we do discuss things amongst ourselves but I wouldn't say we have a specific regular meeting about it.' GP6 (high, over, FH, 3)

'The top 40 give us an indication of where to try next for a prescribing initiative . . the first 5 are impossible to change so you don't.' GP12 (low, over, FH, 3)

'I look at where they have broken it down systemwise sometimes because once we found so much dressings on the PACT ... and we were wondering why this was and I managed to find out the problem.' GP8 (low, under, $N F H, 1)$

'there are reductions in certain areas, the earlier ones, antibiotics which was one of the first that we really concentrated on and a little bit in the GI section as well, the sort of shift from Losec to Zoton for example. So that is starting to show up in PACT.' GP14 (high, under, FH, 7)

- Theme 2 - Ease of understanding:

'Well reasonably easy, the bit I look at, but I generally do not look through it in a great deal of depth.' GP13 (low, over, FH, 3)

'the way they present the data is very difficult.' GP2O (high, under, NFH, 1)

- Theme 3 - Perceptions of the helpfulness of PACT:

'The most important thing is the total prescribing cost ... to see whether we are at least maintaining the level of control on expenditure ... Everybody is having trouble but if we are having no more trouble relative to them then we are not falling behind.' GP12 (low, over, FH, 3)

'I can see if I am sticking up like the BT Tower over the rest of the district or nationally.' GP2O (high, under, $\mathrm{NFH}, 1)$

'Something else that I found very good is that once or twice there has been a few blue pages included and they give a review of some particular topic and they are very good.' GPI (low, over, NFH, 1)

'Did I tell you that PACT data is crap? It's just numbers to me . . all it tells me is whether I am a high prescriber, the average or below... It's a total waste of time as far as I am concerned and I reckon that for most people it's the same in that they do not understand what it is that would bring their prescribing down.' GP18 (high, over, $N F H, 1)$

- Theme 4 - Amount of information in PACT:

'I think that maybe there is a lot of information which I do not think $99 \%$ of people look at ... if it is a slimmer document it is more likely to get read.' GP21 (low, over, FH, 3)

'No [more information], but I'd like someone to sit down with me and tell me where I'm going wrong.' GP19 (low, over, FH, 1)

'it is difficult to criticise something if you do not have a good alternative to suggest ... they need to put it on paper in such a way that at a glance I can see what I am doing right or wrong, I can see my weak areas. ... I would not mind a little appendix of a little bit of written stuff saying 'Do you realise that ... you have written 100 prescriptions for ....' GP2O (high, under, NFH, 1)

${ }^{\text {ahigh }}$ or low $=$ high or low allocated prescribing budget relative to HA average, over or under $=$ overspent or underspent on prescribing budget, $\mathrm{FH}$ or NFH $=$ fundholding or nonfundholding, number $=$ number of partners (practice size) 


\section{How PACT Standard Reports are used}

Many GPs tended to use the data in a limited way and only used a small part of them in an individualistic way. Only a few practices reported using the data in a more systematic way and carrying out a regular analysis of them. This included Practice 10 which employed a pharmacist who carried out this work. However, most GPs said they only 'look' at the data rather than analyse it and use it in a much more informal way.

None of the seven single-handed GPs and few of the small practices said they carried out a regular analysis of the Report, but the GPs in practices with two or more partners reported having some discussion of the data between the partners even if they did not carry out a formal analysis.

There was a wide range of views on the benefits of PACT data but the majority of respondents reported using the information on the front page, which compares total spending per quarter with the $\mathrm{HA}$ and national equivalents and with the same quarter in the previous year. Several also used the comparisons for the individual therapeutic groups. The '20 leading cost drugs' and charts showing trends in prescribing were also cited as sections which were 'looked at'.

Some practices had also used the data in a more specific way to identify problem areas, e.g., dressings, or to monitor the effect of policy decisions and initiatives taken to reduce costs. The sections on '20 leading cost drugs' and 'top $40 \mathrm{BNF}$ sections', in particular, were used to review individual drugs and for monitoring changes.

\section{Ease of understanding of PACT Standard Reports}

There was a very mixed response as to how easy the Standard Report was to understand. Although over half the GPs described the report as easy or reasonably easy to understand, this may have been partly because they only used selected sections of it and did not analyse it in any depth.

Among the GPs who said they did not find it easy, particular difficulties were reported with the bar charts which were felt to be 'slightly confusing' and there was uncertainty whether the data referred to the practice or each partner.

\section{Perception of the helpfulness of PACT data}

The comparison of total costs with HA and national equivalents on the front page was reported to be the most helpful section as this allowed a rapid assessment of their prescribing. There was widespread feeling that a practice should aim for their costs to be near the average, as being too high or too low could draw unwanted attention to their prescribing from the HA.

Many GPs also found it helpful to see trends in their prescribing and the graphs were a useful way of showing these. Several GPs said that they found the bar charts confusing and numbers difficult to interpret whereas the graphs made it easier to see how their prescribing was changing.

A few GPs said that they did not like PACT data or that the data's limitations themselves restricted their usefulness. GP8 suggested that each practice is different and that individuality should be taken into account. GP12 noted that there was no link between prescribing and morbidity. GP18 who had overspent and was on a 'high' budget found the data least helpful.

\section{Amount of information in PACT Standard Report}

Two-thirds of the GPs stated that the amount of information in the Standard Report was 'about right' or 'reasonable' although these included GPs who also said that they only used part of the data. The main reason for not wanting any more information was that there would be insufficient time to read it.

The main request was for more interpretation of the data to be included and for this to be personalized; for example, suggestions for which drugs could be substituted for those highlighted as expensive, key areas for the practice to review and 'how to reduce my costs rather than saying this is what your cost is'.

\section{PACT catalogues}

Only three GPs said that they requested the PACT catalogues. A further eight GPs said that they had requested it in the past, but did not feel the need for it at present, mainly because they would not have time to analyse it. Of the 10 GPs who had not used the PACT catalogue, one said he would like to look at it and another had never heard of it.

\section{Study 2 (questionnaire)}

Response rate and responder characteristics

All 1047 practices in the West Midlands region were mailed and a completed questionnaire was 
returned by 452 practices (43\%). The response rate from larger practices was significantly higher but there was little difference between those who stated that their costs were above or below their HA equivalent (Table 1). The proportion of dispensing practices ( $11.3 \%$ of responding practices) was comparable to the proportion of dispensing GPs $(10.8 \%)$ in the West Midlands. Fifty-five per cent of dispensing practices stated that their prescribing costs were below their HA equivalent.

\section{Use of PACT Standard Reports}

All responders: Of all responders $7 \%$ reported not using any sections of the Standard Report to monitor their costs (Table 2). The mean number of sections used was 5.4 with a median of five. The most frequently used section was the summary information of practice prescribing costs on the front page. Almost a third of respondents did not find any of the sections helpful. Only two sections appeared to be at all helpful for analysing prescribing costs: 20 leading cost drugs and practice prescribing costs.
By fundholding status: A higher percentage of fundholders than nonfundholders used all sections but the difference was only significant for four sections (Table 2), and only two sections were significantly more likely to be considered helpful by fundholders. Fundholders used a median of six sections compared with five sections by nonfundholders $(z=-2.661, P=0.008)$.

By practice size: For all sections of the Standard Report, the proportion of practices using the data varied with practice size (Table 3). Single-handed GPs used PACT data the least and larger practices were almost twice as likely to use some sections. Only two sections showed a significant difference between practice size in how helpful they were felt to be.

By relative costs: In only one section was there a significant difference in the reported use of PACT data between practices with costs above or below their HA equivalent; those below the HA equivalent were more likely to use the section showing average cost per item $(P=0.018)$.

Table 1 Responder characteristics (Study 2 questionnaire) $(n=452)$

Practice size (No. of partners)

Practice list size

Practice location

Fundholding

\section{Dispensing practices}

Practice costs compared with HA equivalent (quarter ending December 1997)

Response rate by practice size (responding practices as a proportion of West Midland practices of that size)

$\begin{array}{lc}\text { mean (SD) } & 3.2(2.0) \\ \text { range } & 1-11 \\ \text { mean (SD) } & 6004(3711) \\ \text { range } & 800-22000 \\ \text { suburban } & 228(51 \%) \\ \text { inner city } & 113(25 \%) \\ \text { rural } & 71(16 \%) \\ \text { combined } & 39(9 \%) \\ \text { Yes } & 245(54 \%) \\ \quad \text { multifund } & 37(8 \%) \\ \quad \text { community } & 18(4 \%) \\ \text { total purchasing pilot } & 13(3 \%) \\ \text { No } & \\ \quad \text { prescribing incentive scheme } & 207(46 \%) \\ & 150(33 \%) \\ \text { below } & 49(11 \%) \\ \text { above } & 243(54 \%) \\ \text { about the same } & 192(43 \%) \\ \text { missing data } & 5(1 \%) \\ 2 & 12(3 \%) \\ 3-4 & 117(32 \%) \\ 5+ & 97(41 \%) \\ & 117(47 \%) \\ & 121(62 \%) \\ & P<0.001\end{array}$


Table 2 Use of PACT data by fundholding status

PACT sections

(listed in order of appearance in Standard

Report)
Total \% (n)

$n=449^{\mathrm{a}}$
Fundholding status

$n=449^{\mathrm{a}}$

\begin{tabular}{lll}
\hline Yes & No & $P$ \\
$n=244$ & $n=205$ & \\
\hline
\end{tabular}

Use

Practice prescribing costs

Practice costs by BNF group

20 leading cost drugs

No. of items practice prescribes

Prescribing by BNF group

Average cost per item

Average cost per BNF group

Graphs of 2 yearly costs

Top 40 BNF sections

Practice profile

Special topics (centre pages)

Did not use

Find most helpful

Practice prescribing costs

Practice costs by BNF group

20 leading cost drugs

No. of items practice prescribes

Prescribing by BNF group

Average cost per item

Average cost per BNF group

Graphs of 2 yearly costs

Top 40 BNF sections

Practice profile

Special topics (centre pages)

No sections useful

PACT catalogue

Sent for PACT catalogue

Used information in catalogue

$86.0(386)$
$62.1(279)$
$73.1(328)$
$49.9(224)$
$35.9(161)$
$55.0(247)$
$29.5(132)$
$36.7(165)$
$25.2(113)$
$53.2(239)$
$37.0(166)$
$6.7(30)$

32.7 (147)

$22.7(102)$

$39.4(177)$

$17.4(78)$

$10.0(45)$

$18.3(82)$

9.6 (43)

10.7 (48)

7.8 (35)

$11.1(50)$

$9.1(41)$

30.5 (137)
87.7 (214)

$67.2(164)$

77.5 (189)

52.0 (127)

38.5 (94)

58.2 (142)

32.4 (79)

42.6 (104)

27.9 (68)

57.8 (141)

38.9 (95)

$6.6(16)$

$32.8(80)$

$25.0(61)$

43.9 (107)

14.8 (36)

11.9 (29)

19.7 (48)

10.2 (25)

13.9 (34)

$9.8(24)$

13.5 (33)

$10.2(25)$

29.1 (71)
$83.9(172)$
$56.1(115)$
$67.8(139)$
$47.3(97)$
$32.7(67)$
$51.2(105)$
$25.9(53)$
$29.8(61)$
$22.0(45)$
$47.8(98)$
$34.6(71)$
$6.8(14)$

32.8 (67)

$20.0(41)$

34.1 (70)

20.5 (42)

$7.8(16)$

16.6 (340)

$8.8(18)$

6.8 (140)

$5.4(11)$

8.3 (17)

$7.8(16)$

32.2 (66)
NS

0.016

0.022

NS

NS

NS

NS

0.005

NS

0.035

NS

NS

NS

NS

0.036

NS

NS

NS

NS

0.015

NS

NS

NS

NS

${ }^{a}$ Missing data three cases

\section{PACT catalogue}

The PACT catalogue was requested by only a third of respondents within the last 2 years (Table 2 ), and slightly fewer had used the information. Larger practices and fundholders were significantly more likely to send for and use a PACT catalogue (Table 3). There was no difference, however, between those practices with costs above or below their HA equivalent. Uses of the catalogue are shown in Table 4 . The catalogue was analysed by the prescribing adviser or a pharmacist in almost a third of the practices who used the data.

\section{Discussion}

This study shows that the majority of the data in the PACT Standard Report was not used by GPs and only simple measures of prescribing were used regularly as a benchmark.

A number of issues identified in the qualitative study were confirmed by the survey of the whole region, for example, that small practices used PACT data differently to larger practices. Some of the single-handed practitioners stated that they knew their own prescribing and therefore had no 
Table 3 Use of PACT data by practice size

PACT sections

(listed in order of appearance in Standard Report)
Practice size (No. of partners)

$\%(n)$

\begin{tabular}{lllll}
\hline 1 & 2 & $3-4$ & $5+$ & $P$ \\
$n=116$ & $n=97$ & $n=117$ & $n=119$ & \\
\hline
\end{tabular}

Use

Practice prescribing costs

Practice costs by BNF group

20 leading cost drugs

No. of items practice prescribes

Prescribing by BNF group

Average cost per item

Average cost per BNF group

Graphs of 2 yearly costs

Top 40 BNF sections

Practice profile

Special topics (centre pages)

Did not use

Find most helpful

Practice prescribing costs

Practice costs by BNF group

20 leading cost drugs

No. of items practice prescribes

Prescribing by BNF group

Average cost per item

Average cost per BNF group

Graphs of 2 yearly costs

Top 40 BNF sections

Practice profile

Special topics (centre pages)

No sections useful

PACT catalogue

Sent for PACT catalogue

Used information in catalogue

$78.4(91)$
$44.8(52)$
$56.9(66)$
$45.7(53)$
$21.6(25)$
$51.7(60)$
$17.2(20)$
$21.6(25)$
$13.8(16)$
$47.4(55)$
$25.0(29)$
$7.8(9)$

$32.8(38)$

$18.1(21)$

25.9 (30)

$19.8(23)$

$6.9(8)$

$19.8(23)$

$8.6(10)$

$8.6(10)$

$6.9(8)$

14.7 (17)

$11.2(13)$

$36.3(42)$

$10.3(12)$

$7.8(9)$

$85.6(83)$
$54.6(53)$
$64.9(63)$
$50.5(49)$
$23.7(23)$
$56.7(55)$
$26.8(26)$
$32.0(31)$
$16.5(16)$
$52.6(51)$
$32.0(31)$
$8.2(8)$

$33.0(32)$

$21.6(21)$

35.1 (34)

19.6 (19)

$10.3(10)$

17.5 (17)

$8.2(8)$

$10.3(12)$

4.1 (4)

10.3 (12)

1.0 (1)

30.9 (30)

$91.5(107)$
$68.4(80)$
$85.5(100)$
$54.7(64)$
$47.0(55)$
$61.5(72)$
$39.3(46)$
$46.2(54)$
$37.6(44)$
$59.8(70)$
$44.4(52)$
$5.1(6)$

33.0 (39)

23.1 (27)

50.4 (59)

19.7 (23)

$13.7(16)$

19.7 (23)

11.1 (13)

15.4 (18)

$10.3(12)$

$12.0(14)$

13.7 (16)

24.8 (29)

$\begin{array}{cc}88.2(105) & 0.031 \\ 79.0(94) & <0.001 \\ 83.2(99) & <0.001 \\ 48.7(58) & \text { NS } \\ 48.7(58) & <0.001 \\ 50.4(60) & \text { NS } \\ 33.6(40) & 0.002 \\ 46.2(54) & <0.001 \\ 31.1(37) & <0.001 \\ 52.9(63) & \text { NS } \\ 45.4(54) & 0.002 \\ 5.9(7) & \text { NS }\end{array}$

31.9 (38)

27.7 (33)

45.4 (54)

10.9 (13)

$9.2(11)$

16.0 (19)

$10.1(12)$

8.4 (10)

$9.2(11)$

$7.6(9)$

$9.2(11)$

$30.3(36)$
NS

NS

0.001

NS

NS

NS

NS

NS

NS

NS

0.011

NS need to analyse it. Small practices were less likely to make use of PACT, i.e., external written information supplied to them about their prescribing behaviour, and this supports other findings from research into the behaviour of small firms (Nayak and Greenfield, 1994).

This study found little difference between practices with high- or low-prescribing costs in the way they used PACT data. However, our finding that GPs with costs below the HA equivalent were more likely to use the section showing average cost per item is consistent with the finding from another study (Avery et al., 2000) that GPs in practices with either high- or low-prescribing costs have different views concerning drug substitution with comparable but cheaper drugs.
GPs receive two different reports detailing their prescribing costs: the PACT Standard Report and a monthly budget statement related to their target budget, and this appears to result in conflicting messages. Many GPs in the interviews reported that they read the 'practice prescribing costs' summary on page one of the Standard Report to see if they were above or below the HA average and used this as a measure of whether their costs were acceptable. This may reflect dissatisfaction with the budget allocation (Baines and Parry, 2000; Roberts, 1998). Some GPs were not motivated to achieve their budget as they considered the budget to be inadequate and unrelated to the needs of their patients. Some expressed concern about the budget-setting process itself, suggesting that if they 
Table 4 Analysis of use of PACT catalogue

\begin{tabular}{ll}
\hline Use described & Practices \\
& $(n=133)$ \\
& $n(\%)$ \\
\hline
\end{tabular}

To review prescribing, e.g., rationalise

$30(22.6)$ from several products to one

To analyse costs

Analysis by practice pharmacist

$20(15.0)$

Analysis by other pharmacist

$20(15.0)$

Focus on specific drug group/items,

$13(9.8)$

$12(9.0)$

e.g., NSAIDS

For use by prescribing adviser

Generic prescribing

$8(6.0)$

Focus on specific conditions, e.g.,

$7(5.3)$

cardiovascular

Develop or review formulary

$7(5.3)$

Audit

$4(3.0)$

Cheaper drugs

3 (2.3)

$3(2.3)$

2 (1.5)

$2(1.5)$

$1(0.8)$

$1(0.8)$

$1(0.8)$

Teaching registrars

Computer analysis by 'Enigma'

Other, e.g., interest, for information

Did not use - too much work

$8(6.0)$

$8(6.0)$

Did not receive, not yet received ing Centre, 1998). It is suggested that this will continue in PCGs (Audit Commission, 2000).

The study raises further issues which are relevant to the management of prescribing in PCGs. PACT is produced nationally by the PPA but suggestions from GPs for additional information were for more personalized information and interpretation. That requires knowledge of the local community and GPs' circumstances and is unlikely to be feasible for the PPA. However, this is an area which PCGs could address at a local level.

Setting appropriate prescribing budgets and helping GPs to understand how they can use available cost data better will be equally important in the PCG climate, since group dynamics within the PCGs could lead to a blame culture directed at overspenders. The continuing development and mergers of PCGs and the opportunity to change to PCT status (Audit Commission, 2000) will emphasize the need for greater understanding of the potential and utility of information systems in order to manage prescribing expenditure most effectively (Majeed and Malcolm, 1999).

\section{Acknowledgements}

This work was funded by the Department of Health R\&D Prescribing Research Programme. We would like to thank Jim Parle and Richard Seal for their help and advice and the GPs who took part in the project.

\section{References}

Audit Commission 2000: The PCG agenda: early progress of primary care groups in 'The New NHS'. London: Audit Commission.

Avery, A.J., Wetzels, R.V., Rodgers, S. and O'Neill, C. 2000: Do GPs working in practice with high or low prescribing costs have different views on prescribing cost issues? British Journal of General Practice 50, 100-4.

Baines, D.L. and Parry, D.J. 2000: Analysis of the ability of the new needs adjustment formula to improve the setting of weighted capitation prescribing budgets in English general practice. British Medical Journal 320, 288-90.

Bateman, D.N., Campbell, M., Donaldson, L.J., Roberts, S.J. and Smith, J.M. 1996: A prescribing incentive scheme for nonfundholding general practices: an observational study. British Medical Journal 313, 535-8.

Bevan, B. 1996: PACT data - avoiding the pitfalls. Pharmaceutical Journal 257, 25-30.

Primary Health Care Research and Development 2002; 3: 105-114 
Bradlow, J. and Coulter A. 1993: Effect of fundholding and indicative prescribing schemes on general practitioners' prescribing costs. British Medical Journal 307, 1186-9.

Bryman, A. and Burgess, R.G., editors, 1994: Analysing qualitative data. London: Routledge.

Greenhalgh, T. 1998: Effective prescribing at practice level should be identified and rewarded. British Medical Journal 316, 750-3.

Majeed, A., Evans, N. and Head, P. 1997: What can PACT tell us about prescribing in general practice? British Medical Journal 315, 1515-9.

Majeed, A. and Head, S. 1998: Capitation based prescribing budgets will not work. British Medical Journal 316, 748-50.

Majeed, A. and Malcolm, L. 1999: Unified budgets for primary care groups. British Medical Journal 318, 772-6.

Mays, N. and Pope, C. 1995: Rigour and qualitative research. British Medical Journal 311, 109-2.

National Prescribing Centre and NHS Executive 1998: GP prescribing support: a resource document and guide for the New NHS. Liverpool: National Prescribing Centre.

Nayak, A. and Greenfield, S. 1994: The use of management accounting information for managing micro businesses. In Hughes, A. and Storey, D., editors, Finance and the small firm. London: Routledge 182-231.

Prescription Pricing Authority 1994: PACT/IPS technical guide. Newcastle: Prescription Pricing Authority.

Prescription Pricing Authority 1997: Annual Report 1996-7. Newcastle: Prescription Pricing Authority.

Roberts, D. 1998: Prescribing allocations: theory into practices. British Journal of General Practice 48, 1463-4.

Ryan, M., Yule, B., Bond, C. and Taylor, R. 1992: Knowledge of drug costs: a comparison of general practitioners in Scotland and England. British Journal of General Practice 42, 6-9.

Stevenson, F.A., Greenfield, S.M., Jones, M., Nayak, A. and Bradley, C.P. 1999: GPs' perception of patient influence on prescribing. Family Practice 16, 255-61. 